\title{
Deletion of the $\delta$ Opioid Receptor Gene Impairs Place Conditioning But Preserves Morphine Reinforcement
}

\author{
Julie Le Merrer, Ainhoa Plaza-Zabala, Carolina Del Boca, Audrey Matifas, Rafael Maldonado, and \\ Brigitte L. Kieffer
}

Background: Converging experimental data indicate that $\delta$ opioid receptors contribute to mediate drug reinforcement processes. Whether their contribution reflects a role in the modulation of drug reward or an implication in conditioned learning, however, has not been explored. In the present study, we investigated the impact of $\delta$ receptor gene knockout on reinforced conditioned learning under several experimental paradigms.

Methods: We assessed the ability of $\delta$ receptor knockout mice to form drug-context associations with either morphine (appetitive)- or lithium (aversive)-induced Pavlovian place conditioning. We also examined the efficiency of morphine to serve as a positive reinforcer in these mice and their motivation to gain drug injections, with operant intravenous self-administration under fixed and progressive ratio schedules and at two different doses.

Results: Mutant mice showed impaired place conditioning in both appetitive and aversive conditions, indicating disrupted context-drug association. In contrast, mutant animals displayed intact acquisition of morphine self-administration and reached breaking-points comparable to control subjects. Thus, reinforcing effects of morphine and motivation to obtain the drug were maintained.

Conclusion: Collectively, the data suggest that $\delta$ receptor activity is not involved in morphine reinforcement but facilitates place conditioning. This study reveals a novel aspect of $\delta$ opioid receptor function in addiction-related behaviors.

Key Words: Instrumental conditioning, knockout mice, lithium, motivation, opiate, reward

W ithin the endogenous opioid system, $\mu$ opioid receptors are necessary for most drugs of abuse to exert their rewarding properties (1). Pharmacological evidence suggests that $\delta$ opioid receptors play a similar role. Delta agonists elicit conditioned place preference (CPP) (2-5), whereas antagonists attenuate CPP to cocaine, methamphetamine, or morphine $(6-8)$ and alter cocaine self-administration (9). In these studies, however, $\delta$ compounds might produce their effects via partial activation of $\mu$ receptors $(10,11)$. Gene knockout therefore represents a unique approach to tackle the role of $\delta$ receptors in vivo. At present however, behavioral characterization of $\delta$ receptor knockout mice $\left(O p r d 1^{-/-}\right)$failed to clarify the implication of $\delta$ receptors in drug reinforcement. Although morphine-induced CPP was decreased in Oprd1 $^{-/-}$mice (6), cannabinoid-induced CPP (12) and morphine self-administration into the ventral tegmental area (13) were preserved.

A major difficulty when assessing drug reinforcement in animal models lies in the tight intertwining of reward and learning processes. Most animal models used to measure rewarding properties of drugs also assess conditioned learning (14), and pharmacological data point toward a role of $\delta$ receptors in learning processes $(15,16)$. Delta receptor blockade or genetic inactivation might cause deleterious effects on learning rather than reward, which might explain the previously observed deficits in drug reinforcement. To challenge this hypothesis, we examined acquisition of either appetitive

From the Département de Neurobiologie et Génétique (JLM, CDB, AM, BLK), Institut de Biologie Moléculaire et Cellulaire, Illkirch, France; and Laboratori de Neurofarmacologia (AP-Z, RM), Departament de Ciències Experimentals i de la Salut, Universitat Pompeu Fabra, Barcelona, Spain.

Address correspondence to Brigitte L. Kieffer, Ph.D., Institut de Biologie Moléculaire et Cellulaire, Département de Neurobiologie et Génétique,

1 rue Laurent Fries, 67404 Illkirch Cedex, France; E-mail: briki@igbmc.fr. Received May 11, 2010; revised Oct 4, 2010; accepted Oct 22, 2010. (morphine-induced) or aversive (lithium-induced) place conditioning in Oprd1 $1^{-1-}$ mice. We assumed that altered opioid reward in mutant mice would specifically result in decreased morphine-induced CPP, whereas a deficit in learning would impact on aversive as well as appetitive conditioning. To further assess $\delta$ receptor contribution to conditioning processes, we tested Oprd $1^{-1-}$ mice for operant morphine self-administration. We evaluated morphine efficiency as a positive reinforcer with a fixed ratio 1 (FR1) schedule of reinforcement and motivation to earn morphine injections under a progressive ratio (PR) schedule.

\section{Methods and Materials}

Independent groups of Oprd1 ${ }^{-1-}$ mice (17) and their wild-type (WT) control subjects were trained for morphine-induced $(5,10,20$ $\mathrm{mg} / \mathrm{kg}, 6$ days) or lithium-induced ( $3 \mathrm{mEq} / \mathrm{kg}, 3$ days) place conditioning and tested either drug-free or under drug effects (statedependency). Other groups of mice were trained to self-administer morphine (.25 or $.5 \mathrm{mg} / \mathrm{kg} /$ infusion) under an FR1 schedule of reinforcement for 10 days. Animals that completed self-administration criteria were moved to a PR schedule of reinforcement on Day 11. The PR session was performed only once and allowed the determination of a breaking-point for each animal (see Supplement 1 for complete protocols and surgical methods).

\section{Results}

\section{Intact Spatial Novelty Discrimination}

We first performed a novelty discrimination test to examine whether mutant mice discriminate spatial cues under the same conditions as for place conditioning. During choice session, WT and Oprd $1^{-1-}$ mice spent more time [novelty: $F(1,20)=6.66, p<.05$ ] and were more active $[F(1,20)=28.81, p<.0001]$ in the novel compartment compared with familiar compartment (Figure 1A). Thus, mice from both lines were similarly able to discriminate between these two environments [gender: $F(1,20)=4.29$ and $F(1,20)=.12$; genotype: $F(1,20)=.06$ and $F(1,20)=.50$, respectively, $p=\mathrm{ns}$ ]. 

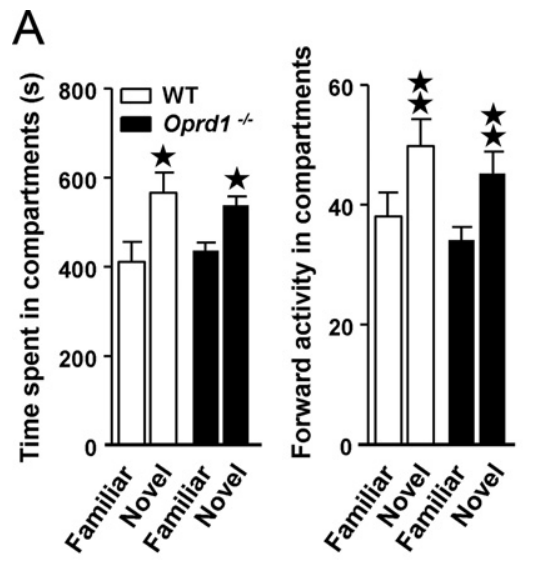

B
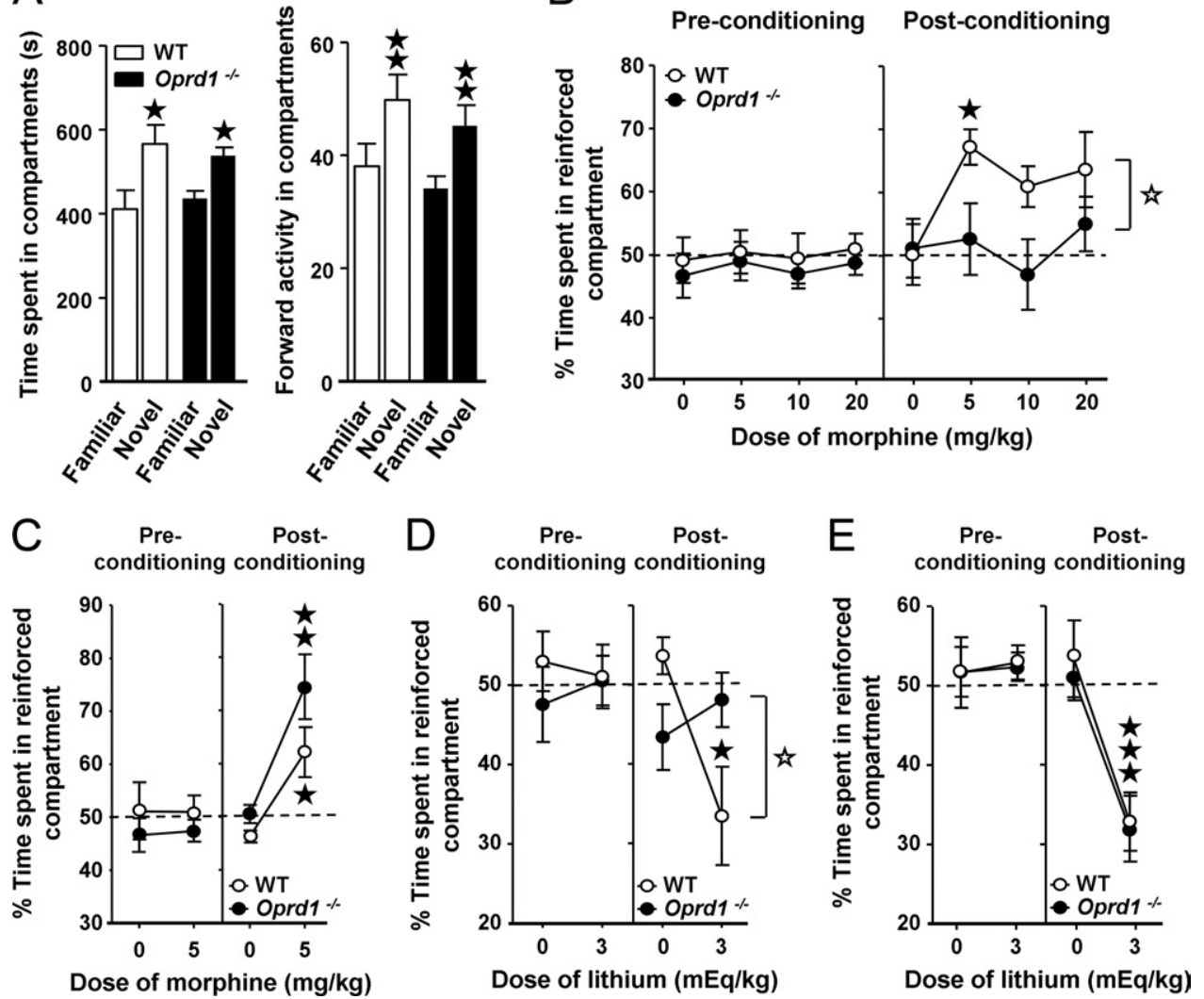

Figure 1. Spatial discrimination and place conditioning in mice lacking the $\delta$ opioid receptor gene (Oprd $1^{-1-}$ ) and their wild-type counterparts (WT). Independent groups of naive animals were used for each set of experiments. (A) The WT and Oprd1 ${ }^{-1-}$ mice spent more time and were more active in the novel versus familiar compartment of the place preference apparatus, showing similar ability to discriminate the two compartments. (B) The WT but not Oprd1 $1^{-1-}$ animals exhibited a significant place preference for the compartment associated with morphine injections over a four-time dose range (5-20 $\mathrm{mg} / \mathrm{kg}$ ) when tested drug-free. (C) The WT and Oprd $1^{-1-}$ animals displayed place preference to morphine $(5 \mathrm{mg} / \mathrm{kg})$ when tested under the effects of the drug. (D) In contrast to WT animals, drug-free Oprd $1^{-1-}$ mice failed to display a place aversion to lithium chloride (3 mEq/kg). (E) When tested under the effect of lithium, WT and mutant animals similarly avoided the lithium-paired compartment. Spatial discrimination data are presented as mean ( \pm SEM) time spent and forward activity (two consecutive beam breaks) in the familiar and the novel compartments ( $n=5-7 /$ gender and genotype). Place preference data show mean ( \pm SEM) time spent in the drug-paired compartment (expressed as a percentage of time spent in the two compartments) during the 20-min pre- and post-conditioning sessions ( $n=3-5 /$ gender, genotype, and dose). 1 solid star $p<.05 ; 2$ solid stars $p<.01 ; 3$ solid stars $p<.001$, comparison with familiar compartment or vehicle control (one-way analysis of variance followed by Dunnett's test where appropriate). 1 open star $p<.05$, comparison between genotypes (four-way analysis of variance).

\section{Decreased CPP to Both Appetitive and Aversive Stimuli}

We submitted Oprd $1^{-1-}$ mice to several place conditioning paradigms. First, distinct groups of animals were tested in a drug-free state after conditioning to saline or morphine (5-20 mg/kg). The WT but not mutant mice acquired morphine CPP [gender: $F(1,48)=.14$, $p=$ ns; genotype: $F(1,48)=5.69, p<.05$; conditioning: $F(1,48)=$ $15.67, p<.001$ ] (Figure 1B). Post hoc comparison with the control group performed for postconditioning session detected a significant CPP at $5 \mathrm{mg} / \mathrm{kg}$ in WT mice. Second, mice were tested under the effect of morphine $(5 \mathrm{mg} / \mathrm{kg})$. Both WT and Oprd1 ${ }^{-/-}$mice displayed similar morphine-induced CPP [gender: $F(1,24)=.36, p=$ ns; genotype: $F(1,24)=2.11, p=$ ns; treatment: $F(1,24)=18.00, p<$ .001 ; conditioning: $F(1,24)=16.26, p<.001$; treatment $\times$ conditioning: $F(1,24)=17.95, p<.001$ ] (Figure 1C). State-dependent morphine-induced CPP was not observed in mice lacking the $\mu$ opioid receptor (Figure S2 in Supplement 1). Third, mice were tested in a drug-free state for lithium-induced conditioned place aversion (CPA). As for morphine CPP, WT but not Oprd $1^{-/-}$animals avoided the lithium-paired compartment [gender: $F(1,26)=.02$, $p=\mathrm{ns}$; conditioning: $F(1,26)=5.79, p<.05$; genotype $\times$ treatment: $F(1,26)=5.52, p<.05$; genotype $\times$ treatment $\times$ conditioning:
$F(1,26)=4.39, p<.05$ ] (Figure 1D). In contrast, when tested under the effects of lithium, WT and Oprd1 $1^{-1-}$ mice displayed similar strong aversion for the lithium-paired compartment [gender: $F(1,26)=2.30, p=\mathrm{ns}$; genotype: $F(1,26)=.09, p=\mathrm{ns}$; treatment: $F(1,26)=13.76, p<.001$; conditioning: $F(1,26)=18.28, p<.001$; treatment $\times$ conditioning: $F(1,26)=19.92, p<.001$ ] (Figure 1E). Altogether the data indicate that both morphine CPP and lithium CPA are impaired in Oprd1 $1^{-/-}$mice.

\section{Maintained Acquisition of Intravenous Morphine Self-Administration and Motivation to Earn Morphine}

We evaluated acquisition of operant morphine self-administration and motivation to obtain the drug at two doses (.25 and .5 $\mathrm{mg} / \mathrm{kg} /$ infusion) in Oprd1 ${ }^{-1-}$ and WT mice. Under an FR1 schedule of reinforcement, a similar percentage of WT and mutant mice reached the acquisition criteria for self-administration at the two doses tested $\left(.25 \mathrm{mg} / \mathrm{kg}: 55 \%\right.$ and $50 \%$, respectively, $\chi^{2}=.125, p=$ $\mathrm{ns} ; .5 \mathrm{mg} / \mathrm{kg}$ : $66.7 \%$ and $53.8 \%$ respectively, $\chi^{2}=.99, p=\mathrm{ns}$ ). Five-way analysis of variance revealed no effect of gender $[F(1,36)=.70, p=\mathrm{ns}]$ or genotype $[F(1,36)=.54, p=\mathrm{ns}]$ but significant discrimination between active and inactive hole 
A
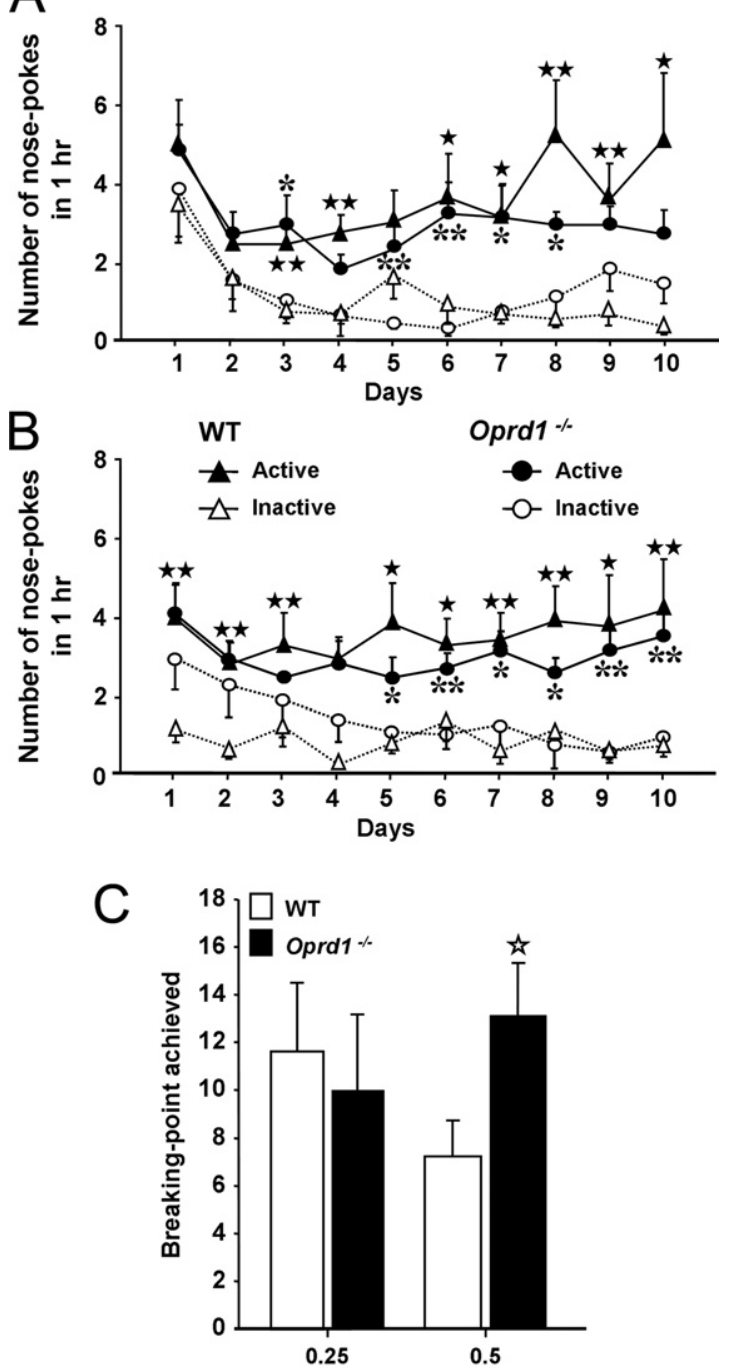

Dose of morphine (mg/kg/infusion)

Figure 2. Operant morphine self-administration in mice lacking the $\delta$ opioid receptor gene (Oprd1 $1^{-1-}$ ) and their wild-type counterparts (WT). Mice from WT and $O p r d 1^{-1-}$ lines discriminate between the active and the inactive holes and acquire self-administration for intravenous morphine at (A) .25 or (B) $.5 \mathrm{mg} / \mathrm{kg} / \mathrm{infusion}$. The data represent the number of nose-pokes in each hole/1-hour daily session. Data are expressed as mean \pm SEM (.25 mg/kg/infusion: $n=3-7 /$ gender and genotype; $.5 \mathrm{mg} /$ $\mathrm{kg} /$ infusion: $n=6-10 /$ gender and genotype). 1 solid star $/{ }^{*} p<.05$; 2 solid stars $/ * * p<.01$, comparison between holes (one-way analysis of variance). (C) The Oprd1 ${ }^{-1-}$ mice achieved a similar breaking-point as WT mice under a progressive ratio schedule of reinforcement. Data analysis at each dose separately shows increased breaking-point at the high dose only, 1 open star $p<.05$, comparison between genotypes (one-way analysis of variance).

$[F(1,36)=36.63, p<.0001]$, significant effect of sessions $[F(9,324)=$ $6.92, p<.0001]$, and significant hole $\times$ session interaction $[F(9,324)=$ $2.39, p<.05]$. Therefore, WT and Oprd $1^{-1-}$ mice similarly acquired operant self-administration for morphine (Figures 2A and 2B). Under a PR schedule, Oprd $1^{-/-}$mice achieved a breaking-point similar to WT mice [genotype: $F(1,21)=.85, p=\mathrm{ns}$; dose: $F(1,21)=.07, p=$ $n s]$ (Figure 2C). These data indicate that acquisition of morphine self-administration and motivation for the drug are preserved in mutant mice.

\section{Discussion}

In our study, Oprd1 ${ }^{-1-}$ mice failed to express morphine CPP over a $5-20-\mathrm{mg} / \mathrm{kg}$ dose range. Mutant animals as WT mice, however, were able to discriminate novel from familiar compartment in the place conditioning apparatus. This suggests that decreased morphine CPP in mutant mice does not result from altered recognition of spatial contexts, at least in the CPP apparatus. Furthermore, when tested under the effects of morphine, Oprd $1^{-/}$mice displayed preference for the morphine-paired context, similar to WT animals. Morphine-induced CPP was thus state-dependent in Oprd1 $1^{-1-}$ mice. Otherwise, state-dependent morphine-induced CPP was not observed in mice lacking $\mu$ opioid receptors, consistent with the essential role of $\mu$ receptors as the primary target for morphine in vivo. State-dependency qualifies a behavioral response that can only be retrieved when the animal experiences the same (drug) state as during the acquisition of this response (18). Interoceptive drug cues can then function as conditioned stimuli and contribute to contextual information together with external cues. In the present study, Oprd $1^{-/-}$mice and not their WT counterparts needed both internal and external cues to express a morphine-induced CPP. Together, these data suggest that the ability to form drug-context associations and/or retrieve such associations was blunted in Oprd $1^{-/-}$mice.

To challenge this hypothesis, we tested Oprd1 $1^{-/-}$mice in a place aversion-conditioning paradigm with lithium chloride (3 $\mathrm{mEq} / \mathrm{kg}$ ) as a potent nonopioid aversive stimulus. Knockout mice failed to display lithium-induced CPA when tested drug-free but showed strong aversion for the lithium-paired compartment when tested under the effects of lithium. Thus, as for morphine-induced CPP, lithium-induced CPA was state-dependent in Oprd1 ${ }^{-1-}$ mice, a finding that strengthens the hypothesis of deficient drug-context associations. Altogether, impaired appetitive and aversive place conditioning demonstrate that reinforced learning is altered in Oprd $1^{-1-}$ mice, regardless of the affective value of the stimuli. This is consistent with previously reported decreased morphine CPP in Oprd $1^{-1-}$ mice (6) as well as in WT animals injected with $\delta$ antagonists before conditioning sessions $(6,8)$. Notably, such effects were not detected in a cannabinoid CPP paradigm (12). In the latter study, increased number of drug-pairings (five sessions vs. three) could have facilitated drug-context associations.

Our place conditioning data provide evidence for impaired drug-context association but do not exclude that drug reward is also reduced in $\delta$ receptor knockout mice. Hence we further addressed consequences of the gene knockout on opioid conditioned reinforcement with operant morphine self-administration. The WT and $O p r d 1^{-1-}$ mice similarly acquired morphine self-administration and reached the acquisition criteria (FR1). This result is consistent with previous studies showing preserved operant responding for food (19) or intra-ventral tegmental area morphine self-administration (13) in Oprd1 ${ }^{-1-}$ mice. Hence, morphine served as an efficient positive reinforcer in both WT and knockout mice. Moreover, mutant mice achieved a similar or even higher breaking-point than WT control subjects under a PR schedule, strongly suggesting that motivation for morphine reinforcement is preserved in the absence of $\delta$ receptors. This result contrasts with the prevailing view of comparable roles of $\mu$ and $\delta$ opioid receptors in mediating drug reinforcement (see introductory text) and further documents the notion of divergent $\delta$ and $\mu$ receptor activities previously established for emotional responses (17) and motor impulsivity (19).

In conclusion, the present study demonstrates that $\delta$ receptor gene knockout alters place conditioning, whereas morphine reinforcement is maintained. The place conditioning phenotype sug- 
gests that $\delta$ receptor activation might facilitate drug-context associations, an effect that might influence contextual aspects of addiction-related behaviors. Neural mechanisms through which these receptors influence conditioning, however, remain to be elucidated. Remarkably, these receptors are expressed in all key brain regions controlling conditioned learning processes $(1,20)$, where they can fine-tune reinforced memory processes.

This work was supported by the Centre de la Recherche Scientifique, Institut de la Santé et de la Recherche Médicale, Université de Strasbourg; the Spanish "Ministerio de Ciencia e Innovación" (\# SAF200764062); and the Catalan Institution for Research and Advanced Studies Foundation (ICREA Academia-2008). We thank the European Union (GENADDICT/FP6 005166) and the National Institutes of Health (NIAAA AA-16658 and NIDA DA-16768/DA-005010) for financial support. The authors report no biomedical financial interests or potential conflicts of interest.

\section{Supplementary material cited in this article is available online.}

1. Le Merrer J, Becker JA, Befort K, Kieffer BL (2009): Reward processing by the opioid system in the brain. Physiol Rev 89:1379-1412.

2. Longoni R, Cadoni C, Mulas A, Di Chiara G, Spina L (1998): Dopaminedependent behavioural stimulation by non-peptide delta opioids BW373U86 and SNC 80: 2. Place-preference and brain microdialysis studies in rats. Behav Pharmacol 9:9-14.

3. Morales L, Perez-Garcia C, Alguacil LF (2001): Effects of yohimbine on the antinociceptive and place conditioning effects of opioid agonists in rodents. Br J Pharmacol 133:172-178.

4. Shippenberg TS, Bals-Kubik R, Herz A (1987): Motivational properties of opioids: Evidence that an activation of delta-receptors mediates reinforcement processes. Brain Res 436:234-239.

5. Suzuki T, Tsuji M, Mori T, Ikeda H, Misawa M, Nagase H (1997): Involvement of dopamine-dependent and -independent mechanisms in the rewarding effects mediated by delta opioid receptor subtypes in mice. Brain Res 744:327-334.

6. Chefer VI, Shippenberg TS (2009): Augmentation of morphine-induced sensitization but reduction in morphine tolerance and reward in deltaopioid receptor knockout mice. Neuropsychopharmacology 34:887-898.

7. Menkens K, Bilsky EJ, Wild KD, Portoghese PS, Reid LD, Porreca F (1992): Cocaine place preference is blocked by the delta-opioid receptor antagonist, naltrindole. Eur J Pharmacol 219:345-346.
8. Suzuki T, Yoshiike M, Mizoguchi H, Kamei J, Misawa M, Nagase H (1994): Blockade of delta-opioid receptors prevents morphine-induced place preference in mice. Jpn J Pharmacol 66:131-137.

9. Ward SJ, Roberts DC (2007): Microinjection of the delta-opioid receptor selective antagonist naltrindole $5^{\prime}$-isothiocyanate site specifically affects cocaine self-administration in rats responding under a progressive ratio schedule of reinforcement. Behav Brain Res 182:140-144.

10. Hutcheson DM, Matthes HW, Valjent E, Sanchez-Blazquez P, RodriguezDiaz M, Garzon J, et al. (2001): Lack of dependence and rewarding effects of deltorphin II in mu-opioid receptor-deficient mice. Eur J Neurosci 13:153-161.

11. Scherrer G, Befort K, Contet C, Becker J, Matifas A, Kieffer BL (2004): The delta agonists DPDPE and deltorphin II recruit predominantly mu receptors to produce thermal analgesia: A parallel study of mu, delta and combinatorial opioid receptor knockout mice. Eur J Neurosci 19:22392248.

12. Ghozland S, Matthes HW, Simonin F, Filliol D, Kieffer BL, Maldonado R (2002): Motivational effects of cannabinoids are mediated by mu-opioid and kappa-opioid receptors. J Neurosci 22:1146-1154.

13. David V, Matifas A, Gavello-Baudy S, Decorte L, Kieffer BL, Cazala P (2008): Brain regional Fos expression elicited by the activation of mubut not delta-opioid receptors of the ventral tegmental area: Evidence for an implication of the ventral thalamus in opiate reward. Neuropsychopharmacology 33:1746-1759.

14. Stephens DN, Duka T, Crombag HS, Cunningham CL, Heilig M, Crabbe JC (2010): Reward sensitivity: Issues of measurement, and achieving consilience between human and animal phenotypes. Addict Biol 15:145168.

15. Jutkiewicz EM, Rice KC, Woods JH, Winsauer PJ (2003): Effects of the delta-opioid receptor agonist SNC80 on learning relative to its antidepressant-like effects in rats. Behav Pharmacol 14:509-516.

16. Yang S, Kawamura Y, Yoshikawa M (2003): Effect of rubiscolin, a delta opioid peptide derived from rubisco, on memory consolidation. Peptides 24:325-328.

17. Filliol D, Ghozland S, Chluba J, Martin M, Matthes HW, Simonin F, et al. (2000): Mice deficient for delta- and mu-opioid receptors exhibit opposing alterations of emotional responses. Nat Genet 25:195-200.

18. Overton DA (1978): Basic mechanisms of state-dependent learning. Psychopharmacol Bull 14:67-68.

19. Olmstead MC, Ouagazzal AM, Kieffer BL (2009): Mu and delta opioid receptors oppositely regulate motor impulsivity in the signaled nose poke task. PLOS ONE 4:e4410.

20. White NM, McDonald RJ (2002): Multiple parallel memory systems in the brain of the rat. Neurobiol Learn Mem 77:125-184. 